\title{
Phase transitions in the Mitsui model
}

\author{
Yu.I. Dublenych \\ Institute for Condensed Matter Physics of the National Academy of Sciences of Ukraine, \\ 1 Svientsitskii Str., 79011 Lviv, Ukraine
}

Received April 14, 2011, in final form June 14, 2011

\begin{abstract}
In this paper, phase transitions in the Mitsui model without longitudinal field but with a transverse one are investigated in the mean field approximation. The one-to-one correspondence has been established between this model and the two-sublattice Ising-type model with longitudinal and transverse fields. Phase diagrams and diagrams of existence of the ferroelectric phase are constructed. In the case $\Omega=0$ ( $\Omega$ is the transverse field), a simple analytical expression for the tricritical temperature and the condition of existence of the tricritical point are obtained. For $\Omega \neq 0$, systems of equations for the tricritical point and for the condition of its existence are written.
\end{abstract}

Key words: phase transition, ferroelectric phase, Mitsui model, tricritical point

PACS: $64.60 . D e, 64.60 . C n, 77.80 .-e$

\section{Introduction}

The Mitsui model was proposed in 1958 [1] to theoretically explain the ferroelectric properties of the Rochelle salt. In 1971, Žekš, Shukla, and Blinc [2] formulated this model in terms of pseudospins and just in this form it is known at the present time.

For better quantitative description of the Rochelle salt the conventional Mitsui model was modified in many ways. For instance, in reference [3] an additional piezoelectric interaction was included in the Hamiltonian of the model and in references [4] and [5] a transverse field (or tunneling) was included as well. To take into account a realistic structure of Rochelle salt crystal, the four-sublattice Mitsui model was considered [6]. Besides the Rochelle salt, the Mitsui model with transverse field was used for theoretical description of some other ferroelectric compounds, notably, $\mathrm{RbHSO}_{4}\left[\right.$ [7, 8] and $\mathrm{NH}_{4} \mathrm{HSO}_{4}[$ [8] crystals.

In reference [9] the effect of hydrostatic pressure on thermodynamic properties of the Rochelle salt was studied. Hydrostatic pressure makes it possible to change the parameters of the model.

It turns out that the Mitsui model covers not only the ferroelectrics of the order-disorder type but also other physical objects with two-minimum asymmetric potential. For instance, in references [10] and [11] pseudospin-electron models based on the Mitsui model were considered.

Despite a rather wide use of the Mitsui model, there is no detailed analysis of its phase behavior. In reference [12] the diagram of existence of the ferroelectric phase was calculated. However, this diagram is far from being complete. More detailed though still incomplete diagram was obtained in reference [13].

In the present paper, a rigorous and original mathematical investigation of phase transitions in the Mitsui model is proposed. We managed to construct a complete phase diagram of the Mitsui model in the mean field approximation, first without tunneling (or transverse field) and then with nonzero tunneling. For all curves (surfaces) of the diagram the analytical expressions, equations or systems of equations are given. 


\section{Hamiltonian of the Mitsui model in the mean field approximation}

The Hamiltonian of the Mitsui model without external field reads

$$
H=-\frac{1}{2} \sum_{i j} J_{i j}\left(S_{i}^{z A} S_{j}^{z A}+S_{i}^{z B} S_{j}^{z B}\right)-\sum_{i j} K_{i j} S_{i}^{z A} S_{j}^{z B}-\Delta \sum_{i}\left(S_{i}^{z A}-S_{i}^{z B}\right)-\Omega \sum_{i}\left(S_{i}^{x A}+S_{i}^{x B}\right) \text {. }
$$

Here subscripts $A$ and $B$ denote two sublattices, $S_{i}^{\alpha A}$ is $\alpha$-component of the pseudospin on $i$ th site of sublattice $A ; J_{i j}$ and $K_{i j}$ are the interactions between pseudospins of the same sublattice and of different sublattices, respectively, $\Delta$ is the asymmetry of the anharmonic potential; transverse field $\Omega$ describes the tunneling between two wells of the two-minimum potential. The model considered is a lattice one. However, since we use the mean field approximation, we do not need to specify the type of the lattice.

The transformations $S_{i}^{z B} \rightarrow-S_{i}^{z B}, K_{i j} \rightarrow-K_{i j}$ transform Hamiltonian (2.1) into the Hamiltonian of the two-sublattice model with longitudinal field $\Delta$ (the same for both sublattices) and transverse field $\Omega$. Therefore, the two Hamiltonians are equivalent.

In the mean-field approximation, Hamiltonian (2.1) reads

$$
H=\frac{N}{2}\left\{K \eta_{A} \eta_{B}+\frac{J}{2}\left(\eta_{A}^{2}+\eta_{B}^{2}\right)\right\}+\sum_{i}\left(H_{i}^{A}+H_{i}^{B}\right)
$$

where the following notations are introduced:

$$
\begin{gathered}
H_{i}^{A}=-\left(\Delta+K \eta_{B}+J \eta_{A}\right) S_{i}^{z A}-\Omega S_{i}^{x A}, \\
H_{i}^{B}=-\left(-\Delta+K \eta_{A}+J \eta_{B}\right) S_{i}^{z B}-\Omega S_{i}^{x B}, \\
K=\sum_{i} K_{i j}=\sum_{j} K_{i j}, \quad J=\sum_{i} J_{i j}=\sum_{j} J_{i j},
\end{gathered}
$$

$\eta_{A}=\left\langle S_{i}^{z A}\right\rangle$ and $\eta_{B}=\left\langle S_{i}^{z B}\right\rangle$ are the average values of pseudospin on sublattices $A$ and $B$, respectively, $N$ is the total number of pseudospins.

\section{3. $\Omega=0$ case}

\subsection{Free energy, thermodynamic equilibrium conditions and order parameters}

Let us first consider the $\Omega=0$ case. In this case the eigenvalues of Hamiltonians $H_{i}^{A}$ and $H_{i}^{B}$ are as follows:

$$
\begin{array}{ll}
\lambda_{A}=-\frac{1}{2}\left(\Delta+K \eta_{B}+J \eta_{A}\right), & -\lambda_{A} ; \\
\lambda_{B}=-\frac{1}{2}\left(-\Delta+K \eta_{A}+J \eta_{B}\right), & -\lambda_{B} .
\end{array}
$$

The partition function for one unit cell reads

$$
Z_{i}=\left(\mathrm{e}^{-\beta \lambda_{A}}+\mathrm{e}^{\beta \lambda_{A}}\right)\left(\mathrm{e}^{-\beta \lambda_{B}}+\mathrm{e}^{\beta \lambda_{B}}\right) \mathrm{e}^{-\beta\left[K \eta_{A} \eta_{B}+\frac{J}{2}\left(\eta_{A}^{2}+\eta_{B}^{2}\right)\right]} .
$$

The free energy per one unit cell is as follows:

$$
\begin{gathered}
F=-\theta \ln Z_{i}, \\
F=K \eta_{A} \eta_{B}+\frac{J}{2}\left(\eta_{A}^{2}+\eta_{B}^{2}\right)-\theta \ln \left(\mathrm{e}^{-\beta \lambda_{A}}+\mathrm{e}^{\beta \lambda_{A}}\right)-\theta \ln \left(\mathrm{e}^{-\beta \lambda_{B}}+\mathrm{e}^{\beta \lambda_{B}}\right),
\end{gathered}
$$

where $\theta=1 / \beta$ is thermodynamic temperature. From thermodynamic equilibrium conditions

$$
\begin{aligned}
& \left(\frac{\partial F}{\partial \eta_{A}}\right)_{T, \Delta}=0 \\
& \left(\frac{\partial F}{\partial \eta_{B}}\right)_{T, \Delta}=0
\end{aligned}
$$


we obtain the following equations for $\eta_{A}$ and $\eta_{B}$ :

$$
\begin{aligned}
& 2 \eta_{A}=\tanh \left(-\beta \lambda_{A}\right), \\
& 2 \eta_{B}=\tanh \left(-\beta \lambda_{B}\right) .
\end{aligned}
$$

Taking into account equations (3.5), we can rewrite the expression for free energy in the following form:

$$
F=K \eta_{A} \eta_{B}+\frac{J}{2}\left(\eta_{A}^{2}+\eta_{B}^{2}\right)+\frac{\theta}{2} \ln \left[\left(\frac{1}{4}-\eta_{A}^{2}\right)\left(\frac{1}{4}-\eta_{B}^{2}\right)\right] .
$$

The system of equations (3.5) has the solutions of two kinds: for the first ones $\eta_{A}=-\eta_{B}$ (then the system is reduced to one equation only), they exist for arbitrary $\Delta$; for the second ones $\eta_{A} \neq-\eta_{B}$, they exist in a bounded region of values of $\Delta$ and correspond to the ferroelectric phase.

Let us divide the $(K, J)$ plane into eight segments as shown in figure 1 The plots of the free energy as a function of $\Delta$ at zero temperature in the centers of the unit-circle arcs for every segment are shown in figure 2, (It is easy to obtain these plots from equations (3.5) and (3.6) setting $\theta \rightarrow 0$ or $\beta \rightarrow+\infty$.) As one can see from figure 2 , in segments 4 and 5 there are no phase transitions, in segments 6,7 , and 8 there are phase transitions at $\Delta=0$ only. In segment 3 there are only second-order phase transitions that are easy to investigate. The most complicated and interesting picture of phase transitions is observed for segments 1 and 2, therefore we consider only the region $K \geqslant 0, J \geqslant 0$.

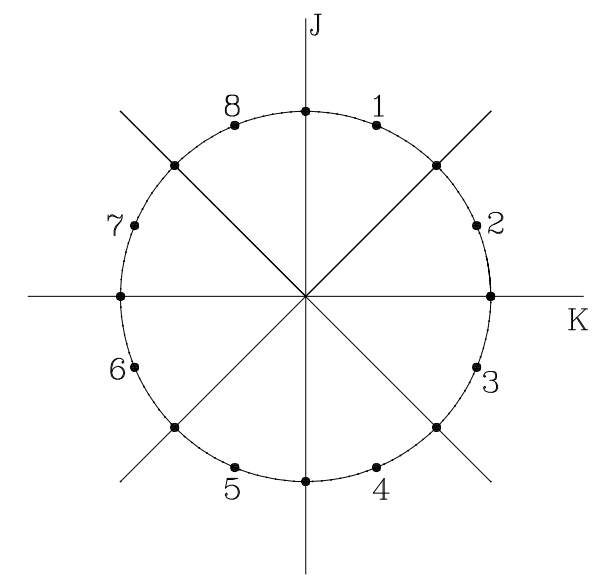

Figure 1. Division of the $(K, J)$ plane into eight segments, which correspond to different relations between parameters $K$ and $J$.

Let us introduce new variables: ferroelectric order parameter $\xi=\eta_{A}+\eta_{B}$ and antiferroelectric order parameter $\sigma=\eta_{A}-\eta_{B}$. Let us also divide all energetic values by $K+J$ and introduce the following notations:

$$
a=\frac{K-J}{K+J}, \quad \gamma=\frac{\Delta}{K+J}, \quad t=\frac{\theta}{K+J}, \quad f=\frac{F}{K+J} .
$$

Now equations (3.5) can be rewritten in the following form:

$$
\begin{aligned}
\mathrm{e}^{\xi / t} & =\frac{(1+\xi)^{2}-\sigma^{2}}{(1-\xi)^{2}-\sigma^{2}} \\
\mathrm{e}^{-a \sigma / t} & =\mathrm{e}^{-2 \gamma / t} \frac{(1+\sigma)^{2}-\xi^{2}}{(1-\sigma)^{2}-\xi^{2}} .
\end{aligned}
$$

If $\xi=0$ (first type solutions, both sublattices are equivalent up to a sign of $\eta$ ) then the first equation becomes an identity and the system (3.8) is reduced to a single equation. If $\xi \neq 0$ (second 

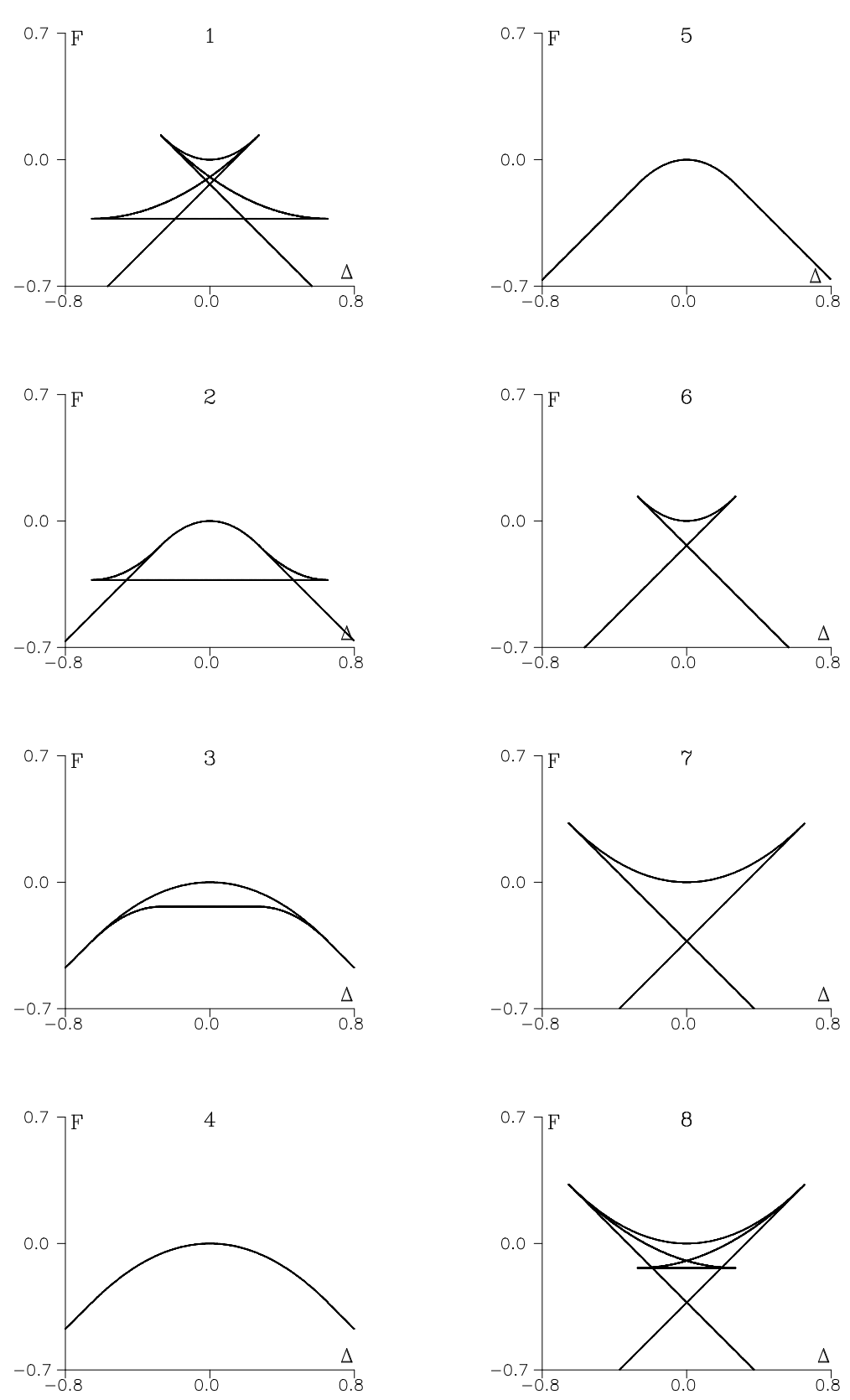

Figure 2. Free energy as a function of $\Delta$ at zero temperature. Numbers over figures correspond to the unit-circle points indicated in figure 1.

type solutions) then equations (3.8) can be rewritten in a simpler form:

$$
\begin{aligned}
& \sigma= \pm \sqrt{1+\xi^{2}+2 \xi \frac{1+\mathrm{e}^{\xi / t}}{1-\mathrm{e}^{\xi / t}}} \\
& \gamma=\frac{a}{2} \sigma+\frac{\xi}{2}+t \ln \frac{1+\sigma-\xi}{1-\sigma+\xi}
\end{aligned}
$$

In view of the symmetry we consider only $\sigma \geqslant 0, \xi \geqslant 0$ and $\gamma \geqslant 0$. 
The expression for the free energy per unit cell in terms of new variables reads

$$
f=\frac{1}{4}\left(\xi^{2}-a \sigma^{2}\right)+\frac{t}{2} \ln \left\{\left[1-(\xi+\sigma)^{2}\right]\left[1-(\xi-\sigma)^{2}\right]\right)-2 t \ln 2 .
$$

\subsection{Second-order phase transitions}

The second-order ferroelectric phase transition corresponds to the branchpoint of the curve $\sigma(\gamma)$ ( $a$ and $t$ being fixed) or of the curve $\sigma(t)$ ( $a$ and $\gamma$ being fixed) where a solution of the first type turns into a solution of the second type. We denote the value of $\sigma$ in this point by $\tilde{\sigma}$. It is as follows:

$$
\tilde{\sigma}=\lim _{\xi \rightarrow 0} \sigma=\sqrt{1-4 t} .
$$

One can see from this expression that the second-order phase transitions exist up to the temperature

$$
t_{\max }=\frac{1}{4}
$$

Substituting $\xi=0$ and $\sigma$ from equation (3.11) in equation (3.9), we obtain the equation for the curve $\gamma(t)$ of the second-order phase transitions:

$$
\gamma=\frac{a}{2} \tilde{\sigma}+t \ln \frac{1+\tilde{\sigma}}{1-\tilde{\sigma}}
$$

Now let us find the minimal temperature for the existence of the second-order phase transitions at fixed $a$. If $a=1$, then only second-order phase transitions exist. If $-1<a<1$, then there are phase transitions of both second and first orders. The latter exist from $t=0$ to a certain value of the temperature. As one can see from figure 3, the tricritical point, i.e. the point where the order of phase transition changes, can be determine from the following condition:

$$
\lim _{\xi \rightarrow 0} \frac{\mathrm{d} \gamma}{\mathrm{d} \sigma}=0
$$

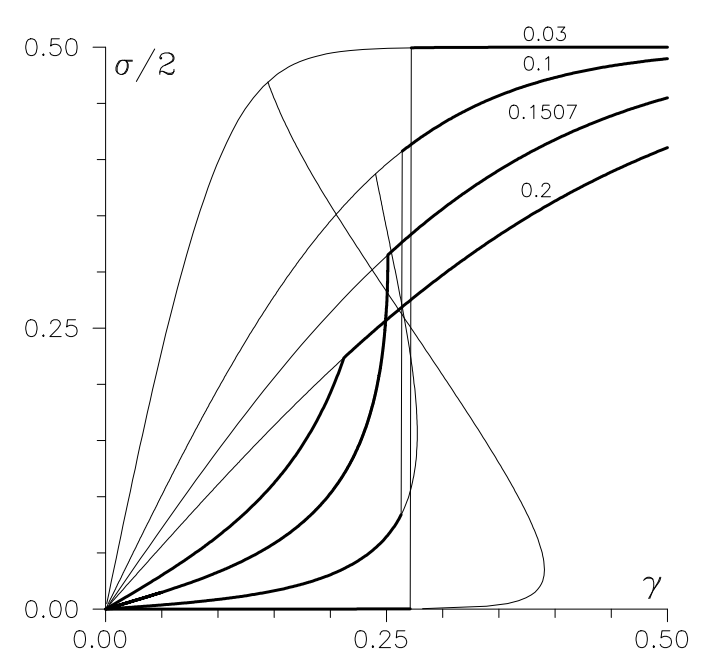

Figure 3. Antiferroelectric order parameter as a function of $\gamma$ for several values of temperature (numbers over the curves). $a=0.0875$. Thermodynamically stable states are depicted by heavy lines. At $t=0.03$ and $t=0.1$ there are first-order phase transitions and at $t=0.1507$ and $t=0.2$ there are second-order ones.

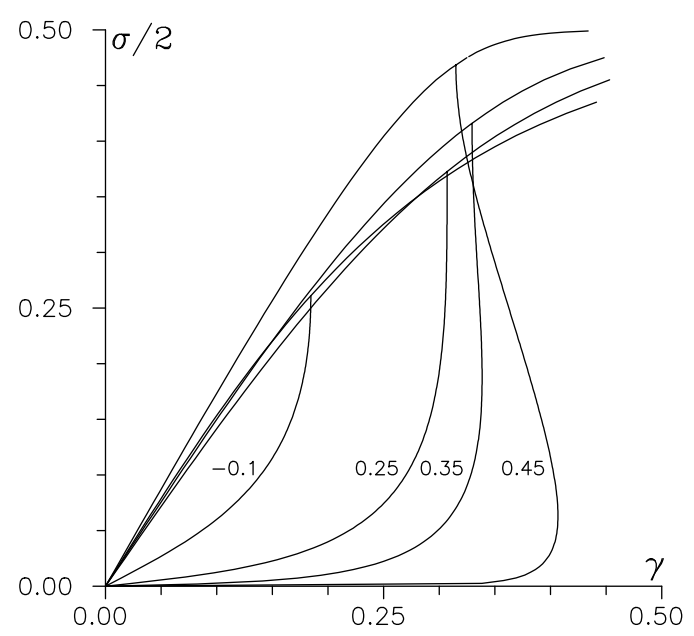

Figure 4. Antiferroelectric order parameter as a function of $\gamma$ for several values of parameter $a$ (numbers near the curves). The values of the temperature are calculated using expression (3.15). At $a=-0.1$ and $a=0.25$ there are second-order phase transitions and at $a=0.35$ and $a=0.45$ there are first-order ones. 
We obtain for the tricritical temperature:

$$
t_{\mathrm{tc}}=\frac{1}{3}+\frac{1}{6(a-1)} .
$$

The tricritical point exists if, at the temperature determined by equation (3.15), the following condition is satisfied:

$$
\lim _{\xi \rightarrow 0} \frac{\mathrm{d}^{2} \gamma}{\mathrm{d} \sigma^{2}} \leqslant 0 \quad(\gamma \geqslant 0) .
$$

This is clear from figure 4 where curves $\sigma(\gamma) / 2$ for several values of parameter $a$ and corresponding values of the temperature [see equation (3.15)] are depicted. The existence of the region where the dependence $\sigma(\gamma)$ for $\xi \neq 0$ is two-valued indicates that there is a first-order phase transition. This two-valuedness disappears with decreasing $a$ and the order of the phase transition changes. From equations (3.15) and (3.16) one obtains:

$$
a \leqslant \frac{1}{4} .
$$

Let us find the maximum of curve $\gamma(t)$ at fixed $a$. The extremum condition $\mathrm{d} \gamma / \mathrm{d} t=0$ and equation (3.13) yield:

$$
\ln \frac{1+\tilde{\sigma}}{1-\tilde{\sigma}}=\frac{a+1}{\tilde{\sigma}}, \quad \gamma=\frac{1}{4}\left(\frac{a+1}{\tilde{\sigma}}+(a-1) \tilde{\sigma}\right) .
$$

Excluding $\tilde{\sigma}$, we obtain the equation for $\gamma$ :

$$
\left(2 \gamma+\sqrt{(2 \gamma)^{2}-a^{2}+1}\right) \tanh \frac{2 \gamma+\sqrt{(2 \gamma)^{2}-a^{2}+1}}{2}=a+1 .
$$

\subsection{First-order phase transitions}

Up to here we analyzed the second-order phase transitions and found the expression for the surface $\gamma=\gamma(t, a)$ of these transitions as well as the tricritical point and the condition for its existence. Now let us consider the first-order phase transitions.

A first-order phase transition corresponds to a point of self-intersection of the curve for the free energy $f(\xi, \sigma)$ as a function of $\gamma$ at fixed $a$ and $t$ (or as a function of $t$ at fixed $a$ and $\gamma$ ). But only this point of self-intersection gives the first-order phase transition in which the multivalued function for the free energy takes the minimal value from all possible values at fixed $\gamma$.

If $-1 \leqslant a \leqslant 1 / 4$, then the first-order phase transitions exists for $0 \leqslant t<t_{\mathrm{tc}}$ [see equation (3.15)]. The curve for them in the $(\gamma, t)$-plain (i.e, the intersection points of the branches $\xi=0$ and $\xi \neq 0$ of the free energy) can be found from the following system of equations:

$$
\begin{aligned}
& \sigma=\sqrt{1+\xi^{2}+2 \xi \frac{1+\mathrm{e}^{\xi / t}}{1-\mathrm{e}^{\xi / t}}} \\
& \gamma=\frac{a}{2} \sigma+\frac{\xi}{2}+t \ln \frac{1+\sigma-\xi}{1-\sigma+\xi} \\
& \gamma=\frac{a}{2} \sigma_{1}+t \ln \frac{1+\sigma_{1}}{1-\sigma_{1}} \\
& f(\xi, \sigma)=f\left(0, \sigma_{1}\right) .
\end{aligned}
$$

If $t=0$, then the first-order phase transition occurs at

$$
\gamma= \pm \frac{1}{4}(a+1) .
$$

In the $t \neq 0$ case the system of equations (3.20) can be solved only numerically. 
The phase coexistence curves for several values of parameter $a$ are shown in figure 5 . The region of ferroelectric phase is bounded by such a curve and by the coordinate axes. The curve of tricritical points [more exactly, its projection on the plane $(\gamma, t)$ ] is also shown in figure 5 (heavy line). From this curve another two curves branch off: the curve of minima of function $\gamma(t)$ for all possible values of $a$ and the curve of maxima for the second-order phase transitions. The curve of tricritical points furcates into the curve of branchpoints and the curve of critical points (dashed line). If $a \gtrsim 0.1793293$ (four curves on the right), then there is a region with two second-order phase transitions (see also figure 6). At this value of $a$ the maximum of the curve $\gamma(t)$ of the second-order phase transitions coincides with the tricritical point.

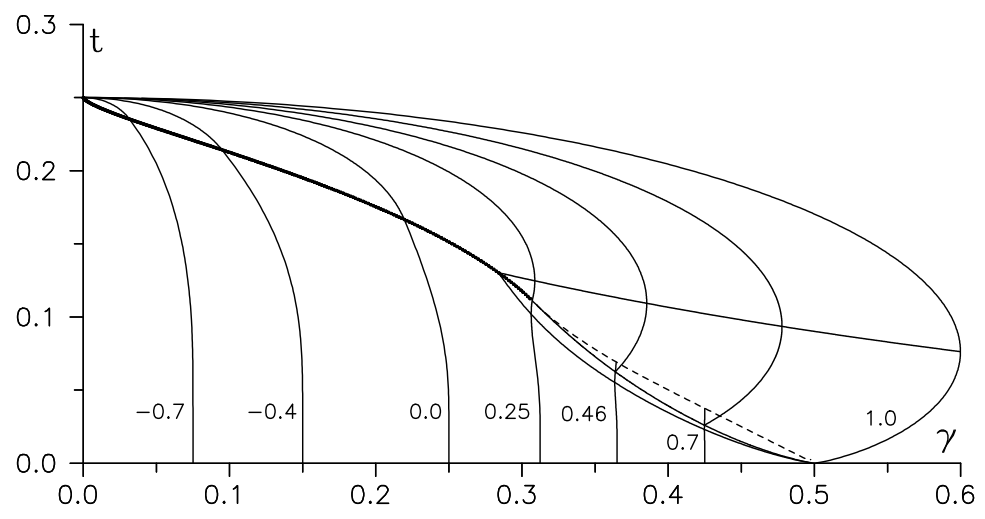

Figure 5. Coexistence curves in the $(\gamma, t)$-plane for several values of parameter $a$ (numbers near the curves). The curve of tricritical points (heavy line), the curve of minima for the first-order phase transitions, the curve of maxima for the second-order phase transitions, the curve of branchpoints and the curve of critical points (dashed line) are indicated.

If $a \geqslant 1 / 4$, then the upper part of the curve of the first-order phase transitions corresponds to the phase transitions within the ferroelectric phase. The temperature of these transitions (at fixed $a$ and $\gamma$ ), i.e. the temperature for the self-intersection points of the free energy curve, can be found from the following system of equations (the solutions of the type $\xi=\xi_{1}$ should be rejected):

$$
\begin{aligned}
\sigma= & \sqrt{1+\xi^{2}+2 \xi \frac{1+\mathrm{e}^{\xi / t}}{1-\mathrm{e}^{\xi / t}}}, \\
\gamma= & \frac{a}{2} \sigma+\frac{\xi}{2}+t \ln \frac{1+\sigma-\xi}{1-\sigma+\xi} \\
\sigma_{1}= & \sqrt{1+\xi_{1}^{2}+2 \xi_{1} \frac{1+\mathrm{e}^{\xi_{1} / t}}{1-\mathrm{e}^{\xi_{1} / t}}}, \\
\gamma= & \frac{a}{2} \sigma_{1}+\frac{\xi_{1}}{2}+t \ln \frac{1+\sigma_{1}-\xi_{1}}{1-\sigma_{1}+\xi_{1}}, \\
& f(\xi, \sigma)=f\left(\xi_{1}, \sigma_{1}\right) .
\end{aligned}
$$

In figures 6(b) and (c) some fragments of phase diagrams in coordinates $(\gamma, t)$ for $a \geqslant 1 / 4$ are shown. The upper part of the curve of the first-order phase transitions corresponds to the transitions within the ferroelectric phase.

If $a \geqslant 1 / 4$, then the critical temperature can be determined from the following system of 

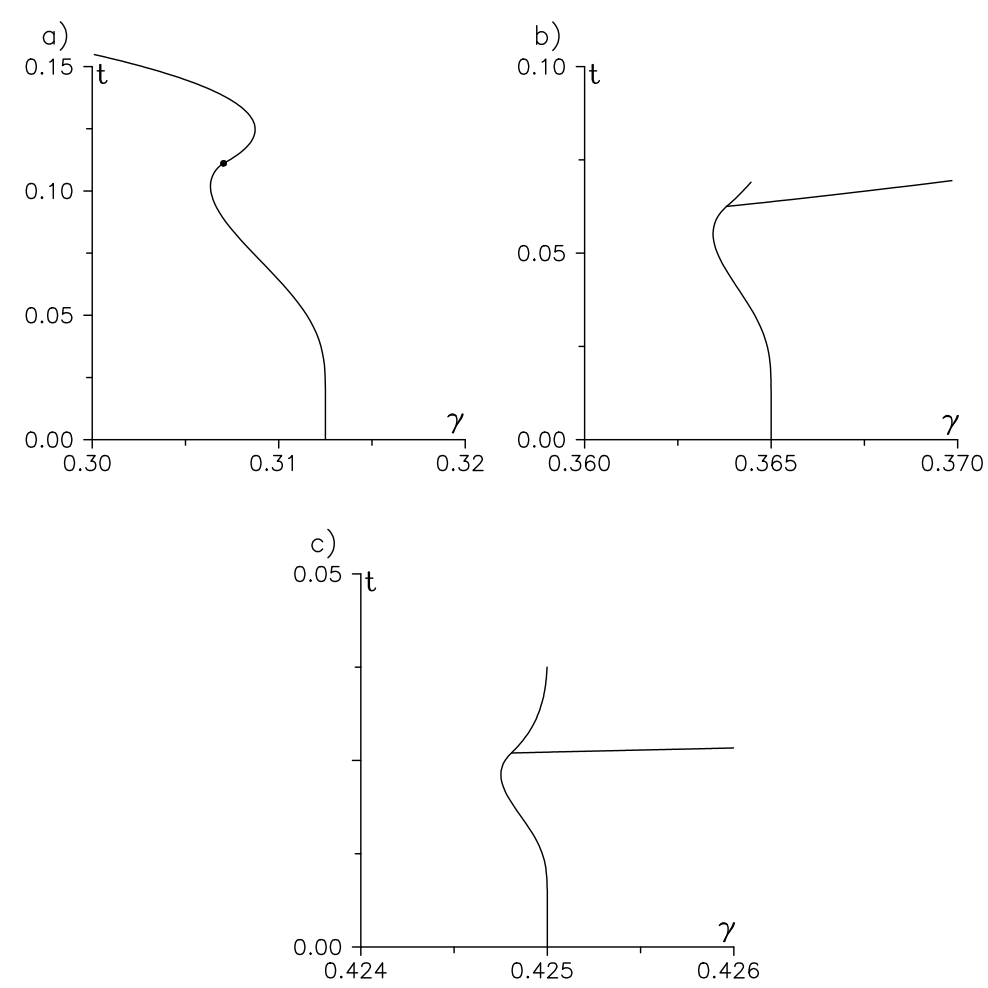

Figure 6. Fragments of the phase coexistence curves for a) $a=0.25$ (the tricritical point is shown), b) $a=0.46$ and c) $a=0.7$ (see figure [5).

equations:

$$
\begin{aligned}
& \frac{\mathrm{d} \gamma}{\mathrm{d} \sigma}=0, \\
& \frac{\mathrm{d}^{2} \gamma}{\mathrm{d} \sigma^{2}}=0, \\
& \sigma=\sqrt{1+\xi^{2}+2 \xi \frac{1+\mathrm{e}^{\xi / t}}{1-\mathrm{e}^{\xi / t}},} \\
& \gamma=\frac{a}{2} \sigma+\frac{\xi}{2}+t \ln \frac{1+\sigma-\xi}{1-\sigma+\xi} .
\end{aligned}
$$

This system of equations can be rewritten in the form:

$$
\begin{aligned}
\sigma^{2} & =1+\xi^{2}-2 t+\frac{2}{a}\left(t-\sqrt{a^{2} \xi^{2}+2 a t \xi^{2}(1-a)+t^{2}(1+a)^{2}}\right) \\
\sigma^{2} & =1+a \xi^{2}-3 t+\frac{1}{a}\left(t-\sqrt{\left[a \xi^{2}(1-a)+t(1+a)\right]^{2}-4 a^{2} \xi^{2}(2 a t-2 t-a)}\right) \\
\sigma^{2} & =1+\xi^{2}+2 \xi \frac{1+\mathrm{e}^{\xi / t}}{1-\mathrm{e}^{\xi / t}}, \\
\gamma & =\frac{a}{2} \sigma+\frac{\xi}{2}+t \ln \frac{1+\sigma-\xi}{1-\sigma+\xi},
\end{aligned}
$$

whence it follows

$$
\xi^{2}=\frac{-q-[(3 a-1) t-a] \sqrt{q-2(a+1) t\left[(a-1)^{2} t-a^{2}\right]}}{2 a(a-1)[2(a-1) t-a]},
$$


where $q=(a-1)\left(3 a^{2}-14 a-1\right) t^{2}-2 a\left(2 a^{2}-5 a+1\right) t+a^{2}(a-1)$, and hence the system of equations is reduced to a single transcendental equation.

As one can see from figure 6 , the curve $\gamma(t)$ of the first-order phase transitions has a minimum if $a$ is big enough. To find it, let us differentiate the last equation of system (3.20) with respect to $t$ and then substitute $\mathrm{d} \gamma / \mathrm{d} t=0$. After simple transformations we obtain the following equation:

$$
\xi^{2}-a \sigma^{2}+4 \gamma \sigma=-a \sigma_{1}^{2}+4 \gamma \sigma_{1},
$$

which together with equations (3.20) gives the point of minimum. At zero temperature the solutions of equations (3.20) also satisfy it:

$$
\lim _{t \rightarrow 0} \frac{\mathrm{d} \gamma}{\mathrm{d} t}=0 .
$$

The branchpoint of the curve (at fixed $a \geqslant 1 / 4$ ) can be found from equations (3.20), substituting

$$
t=\frac{1-\sigma_{1}^{2}}{4} .
$$

\subsection{Regions of existence of the ferroelectric phase}

To conclude, we obtained explicit expressions or system of equations for all special points of the phase curve at fixed $a$. This makes it possible to construct a diagram of the regions where the ferroelectric phase exists. There are seven regions in the $(\gamma, a)$ plane (figures 7 and 8 ). They are bounded by the following curves:

(1) the curve of minima for the first-order phase transitions [equation (3.26)];

(2) the curve of tricritical points [equations (3.11), (3.13), and (3.15)];

(3) the curve of branchpoints [equations (3.20), and (3.28)];

(4) the curve of critical points [equation (3.24)];

(5) the curve of maxima for the second-order phase transitions [equation (3.19)];

(6) the straight line of the first-order phase transitions at zero temperature [equation (3.21)].

In the point $(a \approx 0.179329, \gamma=0.283995)$ the curve of minima for the first-order phase transitions and the curve of maxima for the second-order phase transitions branch off from the

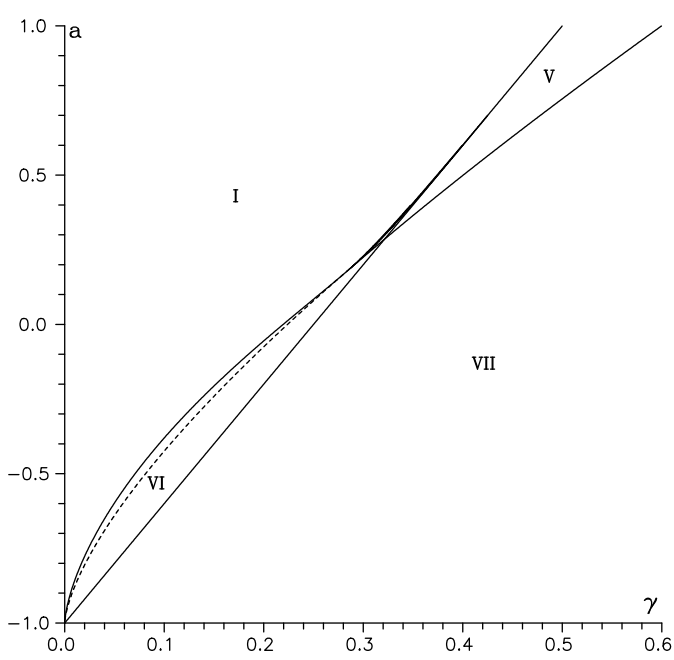

Figure 7. Regions of existence of the ferroelectric phase (see also figures 9 and 10). Dashed line (prolongation of the line which bounds region $\mathrm{V}$ ) is shown only for comparison with the diagram from 12 .

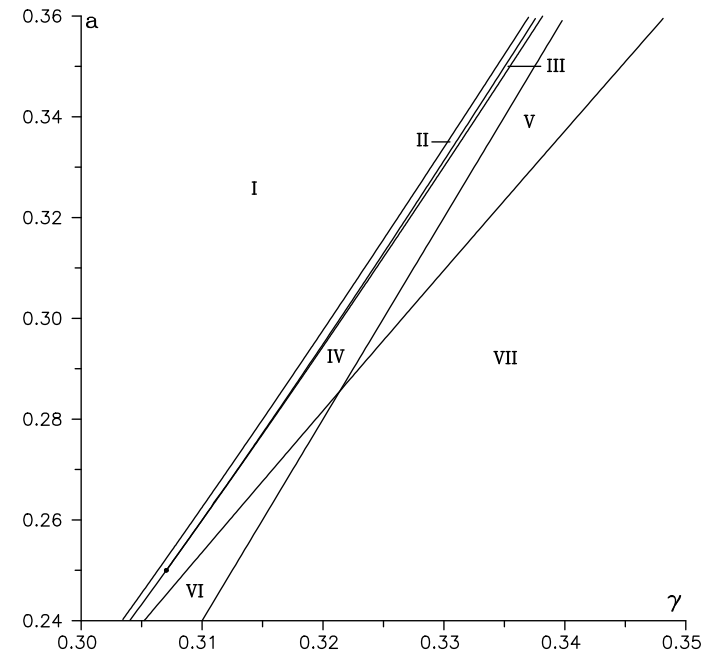

Figure 8. Regions of existence of the ferroelectric phase (fragment). The filled circle is the branchpoint. 
curve of tricritical points and in the point $(a=0.25, \gamma=0.307041)$ the curve of critical points branches out into the curve of branchpoints and the curve of critical points. All curves except for the curve of maxima for the second-order phase transitions converge at $a=1$.
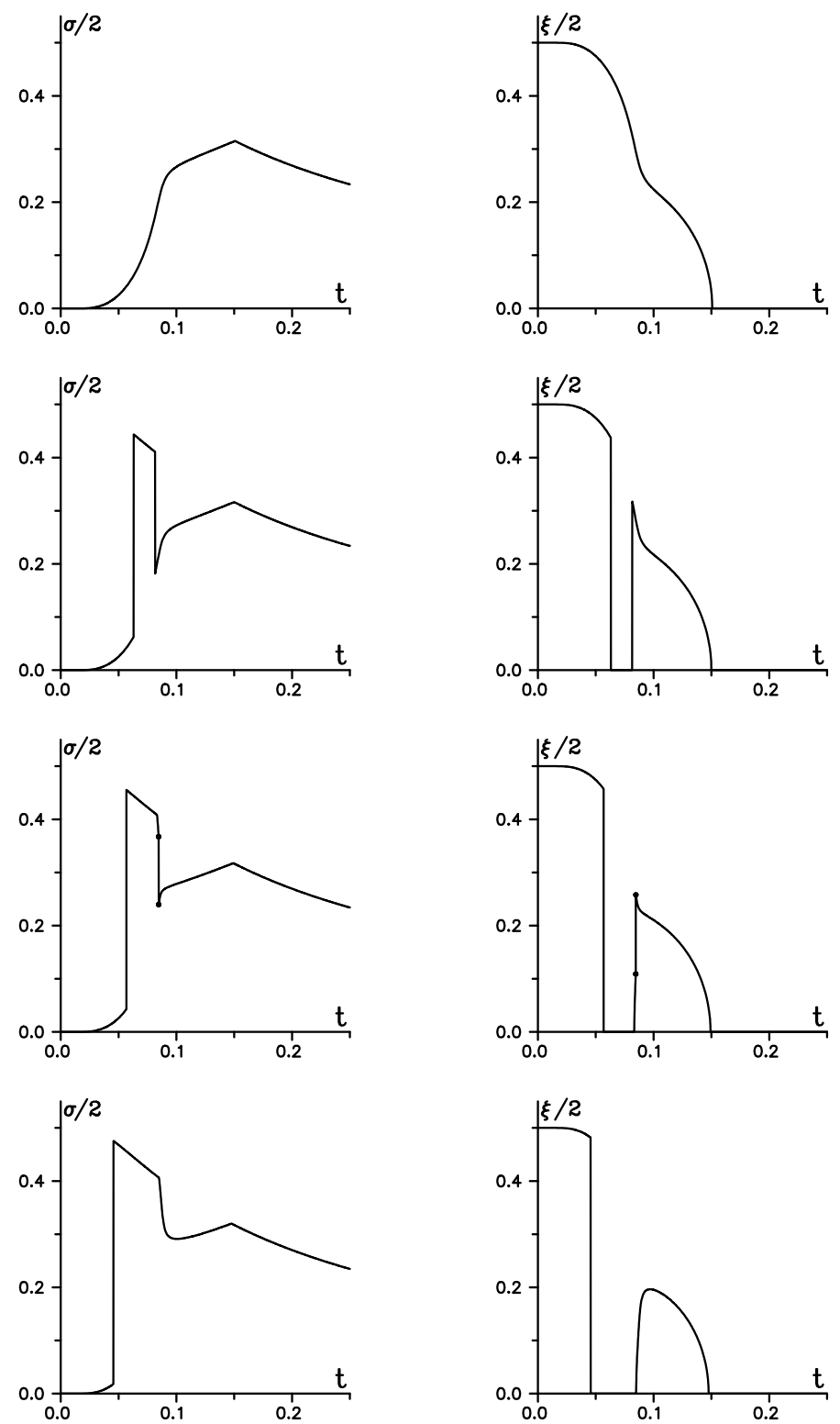

Figure 9. Parameters of ferroelectric and antiferroelectric ordering for different regions where the ferroelectric phase exists. Only thermodynamically stable states are depicted. $a=0.36$. From top to bottom: I) $\gamma=0.337$; II) $\gamma=0.3375$; III) $\gamma=0.338$; and IV) $\gamma=0.339$. For region III the phase transition within the ferroelectric phase is indicated by filled circles.

In figures 9 and 10 the behavior of parameters $\sigma$ and $\xi$ is shown for every region. In region I (figures 7 and 8) only one high-temperature second-order phase transition exists. In region II, in addition to the mentioned one, there are two first-order phase transitions. In narrow region III there are four phase transition: two of the first and two of the second order; one first-order phase transition is within the ferroelectric phase. In region IV there are three phase transitions, one of them being the first-order transition. In region $\mathrm{V}$ there are two second-order phase transitions just like for the Rochelle salt. In region VI there is only one phase transition (of the first order) and in 

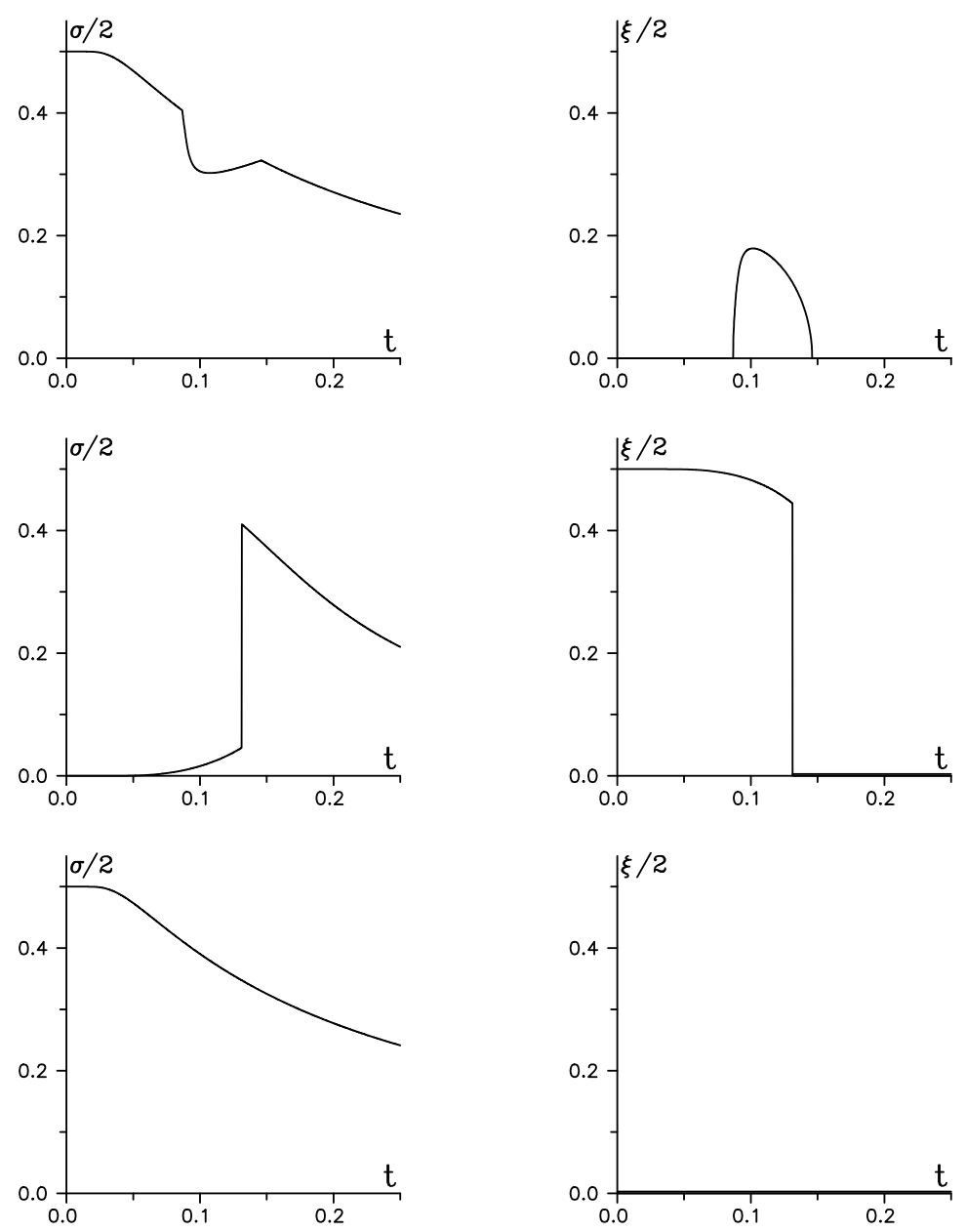

Figure 10. (Continuation of figure 9.) V) $a=0.36, \gamma=0.34$; VI) $a=-0.4, \gamma=0.14$; VII) $a=0.36, \gamma=0.35$.

region VII there are no phase transitions at all.

\section{4. $\Omega \neq 0$ case}

\subsection{Free energy and conditions for thermodynamic equilibrium}

Now let us consider the case of nonzero tunneling. The eigenvalues of one-site Hamiltonians (2.3) and (2.4) are as follows:

$$
\begin{array}{lll}
\lambda_{A}=-\frac{1}{2} \sqrt{\Delta_{A}^{2}+\Omega^{2}}, & -\lambda_{A}, & \Delta_{A}=\Delta+K \eta_{B}+J \eta_{A} ; \\
\lambda_{B}=-\frac{1}{2} \sqrt{\Delta_{B}^{2}+\Omega^{2}}, & -\lambda_{B}, & \Delta_{B}=-\Delta+K \eta_{A}+J \eta_{B} ;
\end{array}
$$

and the free energy per one unit cell reads

$$
\begin{aligned}
F & =K \eta_{A} \eta_{B}+\frac{J}{2}\left(\eta_{A}^{2}+\eta_{B}^{2}\right)-\frac{1}{2}\left(\sqrt{\Delta_{A}^{2}+\Omega^{2}}+\sqrt{\Delta_{B}^{2}+\Omega^{2}}\right)- \\
& -\theta \ln \left(1+\mathrm{e}^{-\beta \sqrt{\Delta_{A}^{2}+\Omega^{2}}}\right)-\theta \ln \left(1+\mathrm{e}^{-\beta \sqrt{\Delta_{B}^{2}+\Omega^{2}}}\right) .
\end{aligned}
$$


The conditions of thermodynamic equilibrium (3.4) yield the following equations:

$$
\begin{aligned}
2 \eta_{A} & =\frac{\Delta_{A}}{\sqrt{\Delta_{A}^{2}+\Omega^{2}}} \tanh \left(-\beta \lambda_{A}\right), \\
2 \eta_{B} & =\frac{\Delta_{B}}{\sqrt{\Delta_{B}^{2}+\Omega^{2}}} \tanh \left(-\beta \lambda_{B}\right) .
\end{aligned}
$$

Like in the $\Omega=0$ case, let us pass to dimensionless values, introducing one more notation:

$$
\omega=\frac{\Omega}{K+J} .
$$

Let us also introduce new variables:

$$
\begin{aligned}
& x=\frac{\Delta_{A}+\Delta_{B}}{2(K+J)}=\frac{\eta_{A}+\eta_{B}}{2}=\frac{\xi}{2}, \\
& y=\frac{\Delta_{A}-\Delta_{B}}{2(K+J)}=\gamma-a \frac{\eta_{A}-\eta_{B}}{2}=\gamma-a \frac{\sigma}{2} .
\end{aligned}
$$

Then the system of equations (4.3) becomes:

$$
\begin{aligned}
& 2 x=A(x+y)-A(-x+y), \\
& \frac{2(\gamma-y)}{a}=A(x+y)+A(-x+y),
\end{aligned}
$$

where

$$
A(z)=\frac{z}{2 \sqrt{z^{2}+\omega^{2}}} \tanh \left(\frac{\sqrt{z^{2}+\omega^{2}}}{2 t}\right),
$$

and the expression for free energy takes the form:

$$
\begin{aligned}
f & =x^{2}-\frac{(\gamma-y)^{2}}{a}-\frac{1}{2}\left(\sqrt{(x+y)^{2}+\omega^{2}}+\sqrt{(x-y)^{2}+\omega^{2}}\right)- \\
& -t \ln \left(1+\mathrm{e}^{-\sqrt{(x+y)^{2}+\omega^{2}} / t}\right)-t \ln \left(1+\mathrm{e}^{-\sqrt{(x-y)^{2}+\omega^{2}} / t}\right) .
\end{aligned}
$$

\subsection{Second-order phase transitions}

Like in the $\Omega=0$ case, the system of equations (4.6) has solutions of two types: 1) $x=0$; then the first equation becomes an identity; and 2) $x \neq 0$, which corresponds to the ferroelectric phase. Letting $x$ tend to zero, we obtain from (4.6) the system of equations for hypersurface $\gamma=\gamma(\omega, a, t)$ of the second-order phase transitions:

$$
\begin{aligned}
& B(\tilde{y})-1=0, \\
& \gamma=\tilde{y}+a A(\tilde{y}),
\end{aligned}
$$

where $B(z)=\mathrm{d} A(z) / \mathrm{d} z, \tilde{y}=\lim _{x \rightarrow 0} y$. Having determined $B(z)$, we can write the system in the form:

$$
\begin{aligned}
& s^{2}-\frac{2 t \omega^{2} s}{\tilde{y}^{2} \sqrt{\tilde{y}^{2}+\omega^{2}}}+\frac{4 t\left(\tilde{y}^{2}+\omega^{2}\right)}{\tilde{y}^{2}}-1=0, \\
& \gamma=\tilde{y}+\frac{a s \tilde{y}}{2 \sqrt{\tilde{y}^{2}+\omega^{2}}},
\end{aligned}
$$

where the following notation is introduced:

$$
s=\tanh \left(\frac{\sqrt{\tilde{y}^{2}+\omega^{2}}}{2 t}\right) .
$$


Setting $\gamma$ equal to zero in equation (4.10), we obtain the expression for maximal temperature at which the phase transitions exist:

$$
t_{\max }=\frac{\omega}{\ln \frac{1+2 \omega}{1-2 \omega}} .
$$

The logarithm in the latter expression makes sense if $\omega \leqslant 1 / 2$. Hence,

$$
\omega_{\max }=\frac{1}{2} .
$$

Differentiating equations (4.10) with respect to $t$, setting $\mathrm{d} \gamma / \mathrm{d} t$ equal to zero, and eliminating $\mathrm{d} \tilde{y} / \mathrm{d} t$, we obtain after simple transformations:

$$
\begin{aligned}
& 2(1+a)\left(\tilde{y}^{2}+\omega^{2}\right)\left(\tilde{y}^{2} s \sqrt{\tilde{y}^{2}+\omega^{2}}-t\left(\tilde{y}^{2}+\omega^{2}\right)\right) \\
& =a\left(3 t \omega^{2} s \sqrt{\tilde{y}^{2}+\omega^{2}}-2 \tilde{y}^{2} s \sqrt{\tilde{y}^{2}+\omega^{2}}+6 t \omega^{2}\left(\tilde{y}^{2}+\omega^{2}\right)-\omega^{2} \tilde{y}^{2} s^{2}\right) .
\end{aligned}
$$

This equation together with equations (4.10) determines the point of maximum for the curve $\gamma(t)$ of the second-order phase transitions (at fixed $\omega$ and $a$ ).

The equation for tricritical point is similar to that in the $\Omega=0$ case:

$$
\lim _{x \rightarrow 0} \frac{\mathrm{d} \gamma}{\mathrm{d} y}=0
$$

From this equation we obtain:

$$
(a+1) D(\tilde{y})-3 a[C(\tilde{y})]^{2}=0,
$$

where the following notations are introduced:

$$
C(z)=\frac{\mathrm{d}^{2} A(z)}{\mathrm{d} z^{2}}, \quad D(z)=\frac{\mathrm{d}^{3} A(z)}{\mathrm{d} z^{3}} .
$$

The tricritical point exists if both equation (4.14) and the following condition are satisfied:

$$
\lim _{x \rightarrow 0} \frac{\mathrm{d}^{2} \gamma}{\mathrm{d} y^{2}} \leqslant 0 \quad(\gamma \geqslant 0) .
$$

This yields the equation

$$
8[D(\tilde{y})]^{3}-9 C(\tilde{y}) D(\tilde{y}) E(\tilde{y})+\frac{9}{5}[C(\tilde{y})]^{2} F(\tilde{y})=0,
$$

where

$$
E(z)=\frac{\mathrm{d}^{4} A(z)}{\mathrm{d} z^{4}}, \quad F(z)=\frac{\mathrm{d}^{5} A(z)}{\mathrm{d} z^{5}} .
$$

If the second-order phase transitions exist until $t=0$, then it follows from equations (4.10), that for this zero-temperature transition $\gamma$ is the following:

$$
\gamma=(2 \omega)^{-2 / 3}\left(a+(2 \omega)^{2 / 3}\right) \tilde{y}
$$

where

$$
\tilde{y}=\frac{1}{2}(2 \omega)^{2 / 3}\left(1-(2 \omega)^{2 / 3}\right)^{1 / 2} .
$$

Rewriting equation (4.15) for zero temperature we obtain:

$$
a=\frac{5(2 \omega)^{2 / 3}-4}{4(2 \omega)^{2 / 3}-5} .
$$

If $a$ is bigger than this value (at fixed $\omega$ ), then the second-order phase transitions begin at zero temperature. It follows from equation (4.18) that equation (4.22) is satisfied under condition

$$
\omega \geqslant 2^{-5 / 2} \approx 0.1768
$$

then $a \leqslant 1 / 2$. 


\subsection{First-order phase transitions}

The points of the first-order ferroelectric phase transitions can be calculated from the following system of equations:

$$
\begin{aligned}
2 x & =A(x+y)-A(-x+y), \\
\frac{2(\gamma-y)}{a} & =A(x+y)+A(-x+y), \\
\frac{\gamma-y_{1}}{a} & =A\left(y_{1}\right), \\
f(x, y) & =f\left(0, y_{1}\right) .
\end{aligned}
$$

Differentiating the last equation with respect to $t$ and eliminating the derivatives, we obtain the following equation:

$$
\begin{aligned}
x^{2} & -\frac{(\gamma-y)^{2}}{a}-\left(x+\frac{\gamma-y}{a}\right) \frac{(x+y)^{2}+\omega^{2}}{x+y} \\
& -\left(x-\frac{\gamma-y}{a}\right) \frac{(x-y)^{2}+\omega^{2}}{x-y}=-\frac{\left(\gamma-y_{1}\right)^{2}}{a}-\frac{2\left(\gamma-y_{1}\right)}{a} \frac{y_{1}^{2}+\omega^{2}}{y_{1}},
\end{aligned}
$$

which, together with the system of equations (4.24), yields the extremum of the curve $\gamma(t)$ of the first-order phase transitions (at fixed $\omega$ ). If $t=0$, then the solutions of the system of equations (4.24) satisfy equation (4.25) as well. Hence, for the curve $\gamma(t)$ of the first-order phase transitions we have

$$
\lim _{t \rightarrow 0} \frac{\mathrm{d} \gamma}{\mathrm{d} t}=0
$$

Like in the $\Omega=0$ case, we can obtain the system of equations for the tricritical point setting $\mathrm{d} \gamma / \mathrm{d} y$ and $\mathrm{d}^{2} \gamma / \mathrm{d} y^{2}$ equal to zero:

$$
\begin{aligned}
& a=\frac{2-B_{-}-B_{+}}{2 B_{-} B_{+}-B_{-}-B_{+}}, \\
& C_{+}\left(1-B_{-}\right)^{3}+C_{-}\left(1-B_{+}\right)^{3}=0, \\
& 2 x=A_{+}-A_{-}, \\
& \frac{2(\gamma-y)}{a}=A_{+}+A_{-},
\end{aligned}
$$

where $A_{ \pm}=A( \pm x+y), B_{ \pm}=B( \pm x+y)$, and $C_{ \pm}=C( \pm x+y)$.

Rewriting the system of equations (4.27) for zero temperature, we obtain:

$$
\begin{aligned}
& \frac{z_{+}\left(2\left(z_{-}^{2}+\omega^{2}\right)^{3 / 2}-\omega^{2}\right)^{3}}{\left(z_{-}^{2}+\omega^{2}\right)^{2}}+\frac{z_{-}\left(2\left(z_{+}^{2}+\omega^{2}\right)^{3 / 2}-\omega^{2}\right)^{3}}{\left(z_{+}^{2}+\omega^{2}\right)^{2}}=0 \\
& \frac{z_{+}}{2 \sqrt{z_{+}^{2}+\omega^{2}}}-\frac{z_{-}}{2 \sqrt{z_{-}^{2}+\omega^{2}}}-z_{+}+z_{-}=0 \\
& a=\frac{4\left(z_{+}^{2}+\omega^{2}\right)^{\frac{3}{2}}\left(z_{-}^{2}+\omega^{2}\right)^{\frac{3}{2}}-\omega^{4}}{\omega^{2}\left(\omega^{2}-\left(z_{+}^{2}+\omega^{2}\right)^{\frac{3}{2}}-\left(z_{-}^{2}+\omega^{2}\right)^{\frac{3}{2}}\right)}+1 \\
& \gamma=\frac{a}{4}\left(\frac{z_{+}}{\sqrt{z_{+}^{2}+\omega^{2}}}+\frac{z_{-}}{\sqrt{z_{-}^{2}+\omega^{2}}}\right)+\frac{1}{2}\left(z_{+}+z_{-}\right)
\end{aligned}
$$

where $z_{ \pm}= \pm x+y$. The nontrivial solution of this system at fixed $\omega \leqslant 2^{-5 / 2}$ corresponds to the point where the curve of the first-order zero temperature phase transitions and the curve of the critical points end. 


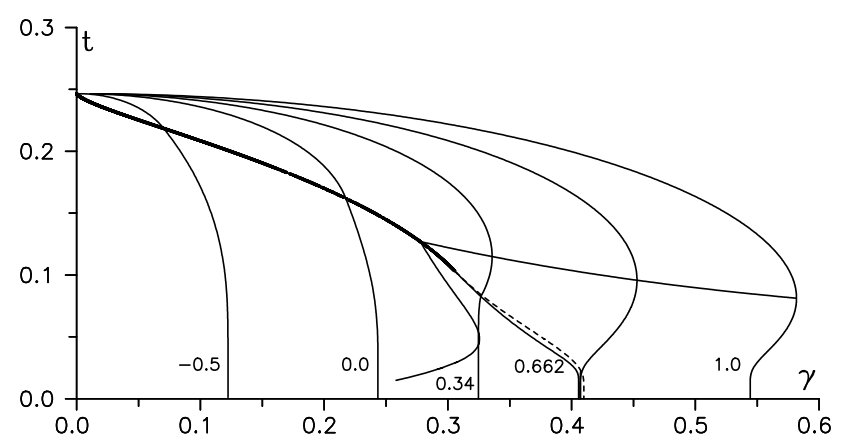

Figure 11. Phase coexistence curves in $(\gamma, t)$-plane for several values of $a$ (number near curves), curve of tricritical points (hard line), curve of extrema for the first-order phase transitions (not hole), curve of maxima for the second-order phase transitions, curve of branchpoints and curve of critical points (dashed line). $\omega=0.1$.

The phase coexistence curves in the $(\gamma, t)$-plane for several values of $a$ as well as the curves of tricritical, critical, and branchpoints at $\omega=0.1$ are depicted in figure 11. The latter two curves do not converge at zero temperature and, hence, there is an interval of values of $a$ where the phase diagrams look as shown in figure 12 (b), i.e., they are composed of the curve of second-order phase transitions and of the curve of first order phase transitions within ferroelectric phase. At $\omega=2^{-5 / 2}$ the curves of critical points and branch- points converge again at zero temperature, and if $2^{-5 / 2}<\omega<0.196815$ these curves converge at nonzero temperatures and pass into the curve

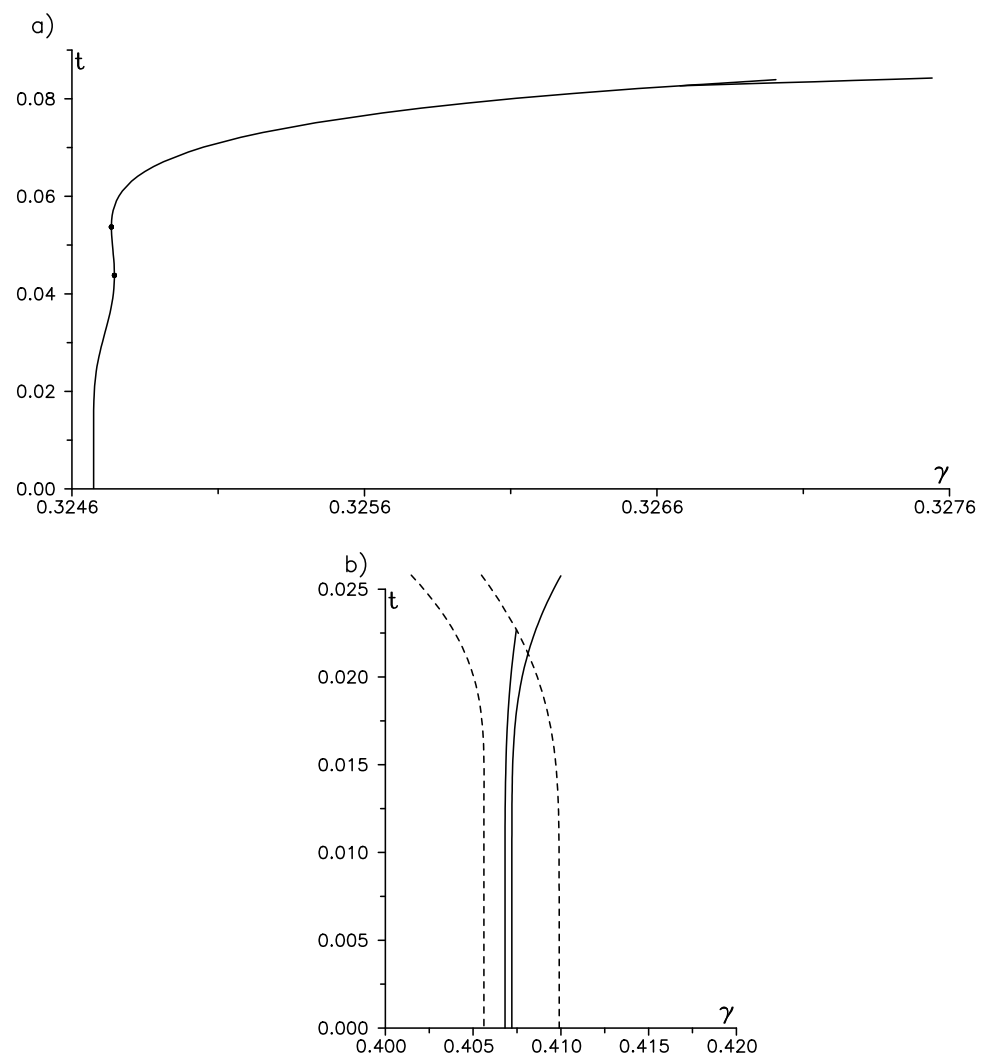

Figure 12. Fragments of the phase coexistence curves at $\omega=0.1$ (see figure 11). a) $a=0.34$ (circles are the extremum points), b) $a=0.662$ (dashed line on the left is the curve of branchpoints and the line on the right is the curve of critical points). 
of tricritical points (figure 13). The latter is many-valued in low-temperature region. This leads to the existence of a new type of phase diagram with two tricritical points (figure 14). If $\omega$ is bigger than $\approx 0.196815$, only the curve of tricritical points remains.

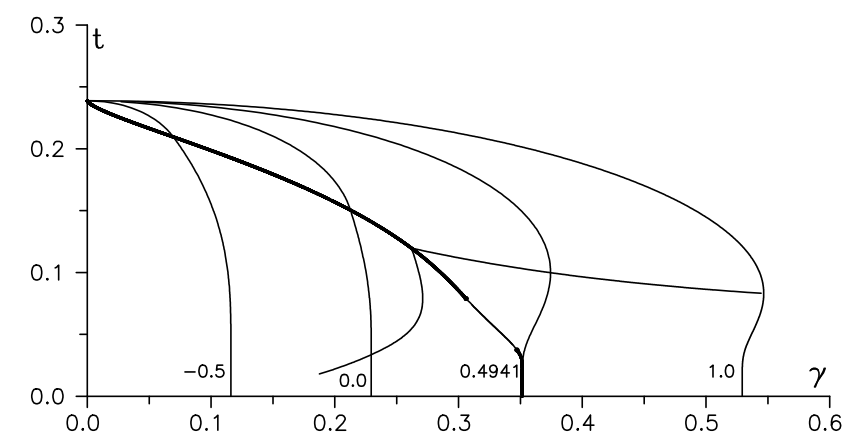

Figure 13. Phase coexistence curves in $(\gamma, t)$-plane for several values of $a$ (number near curves). $\omega=0.18$. The heavy line is the line of tricritical points. The curve of tricritical points and the curve of branchpoints are indistinguishable on this scale.

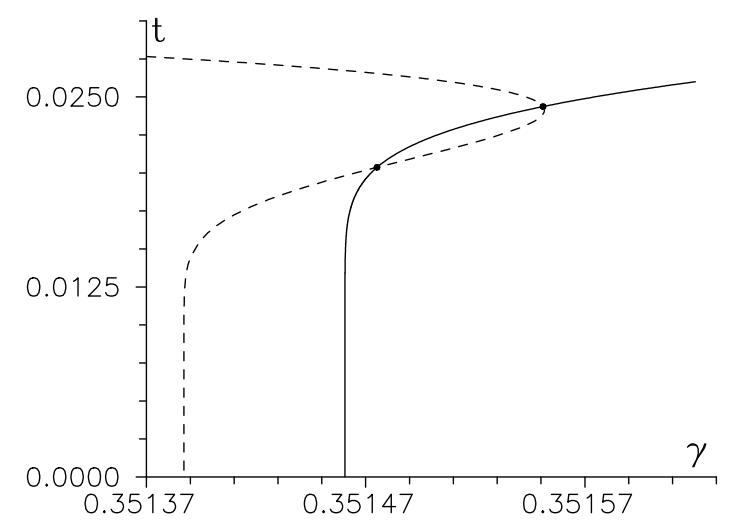

Figure 14. Fragment of the phase coexistence curve at $a=0.4941, \omega=0.18$ (see figure 13). Dashed line is the curve of tricritical points.

\subsection{Regions of existence of the ferroelectric phase}

In figures 15, 20, the diagram of the regions where the ferroelectric phase exists is shown for $\omega=0.1$. The diagram is composed of the same curves as in the $\Omega=0$ case as well as of the straight line of second-order zero-temperature phase transitions (4.20) and the curve of maxima for the first-order phase transitions (4.25) which passes very closely to the curve of first-order zero-temperature phase transitions.

Like in the $\omega=0$ case, two curves branch off (in the same point) from the curve of tricritical points: the curve of minima for the first-order phase transitions and the curve of maxima for the second-order phase transitions. If $\omega<0.196815$, then the curve of tricritical points bifurcates in the curve of critical point and the curve of branchpoints which, if $\omega>2^{-5 / 2}$, conflow again into the curve of tricritical points. The curve of first-order zero-temperature phase transitions is composed of two parts. The first one corresponds to the transitions from (or into) the ferroelectric phase. It begins at the origin of coordinates and ends at an extremity of the curve of the branchpoints $\left(\omega \leqslant 2^{-5 / 2}\right)$ or of the tricritical points $\left(\omega>2^{-5 / 2}\right)$ (this extremity is an end point of the curve for the second-order zero-temperature phase transitions). The second part exists only at $\omega<2^{-5 / 2}$, corresponds to the phase transitions within the ferroelectric phase and ends at an extremity of the curve of the critical points (see figure 16). In the $\omega \geqslant 2^{-5 / 2}$ case, the point where the curve 


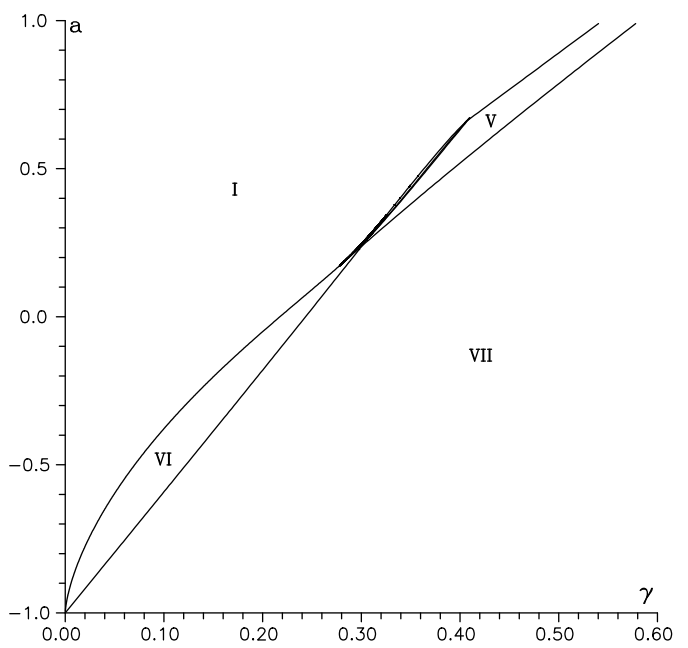

Figure 15. Regions of existence of the ferroelectric phase. $\omega=0.1$.

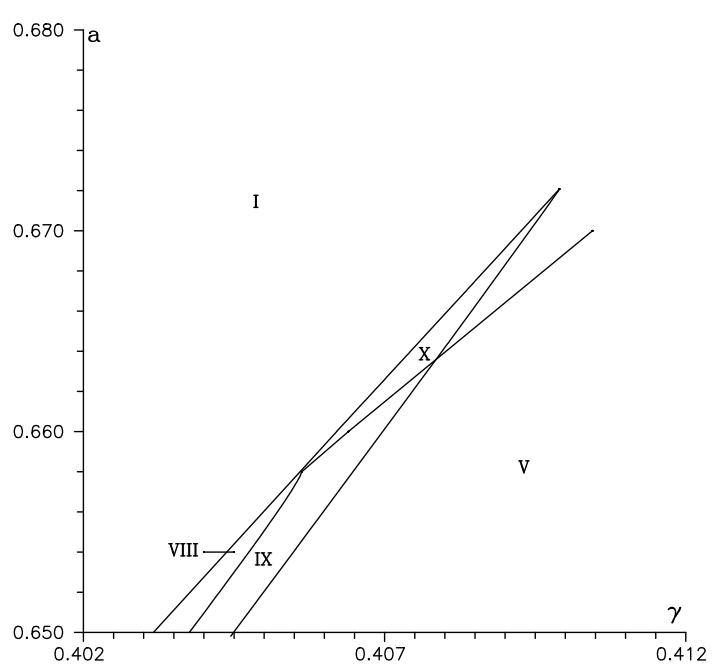

Figure 16. Regions of existence of the ferroelectric phase (fragment). $\omega=0.1$. (See also figure 21).

of tricritical points and the curve of the first-order zero-temperature phase transitions meet is determined by equation (4.22); in this point the latter curve smoothly turns into the curve of the second-order zero-temperature phase transitions.

At nonzero $\omega$ new regions occur. For instance, in region VIII there are one low-temperature first-order phase transition into the ferroelectric phase and one second-order phase transition. In region IX three phase transitions exist: first-order one and two second-order ones (the first-order phase transition occurs within the ferroelectric phase). In region $\mathrm{X}$ there are one first-order phase transition within the ferroelectric phase and one second-order phase transition.

The curves of minima and maxima for the first-order phase transitions converge in region VIII

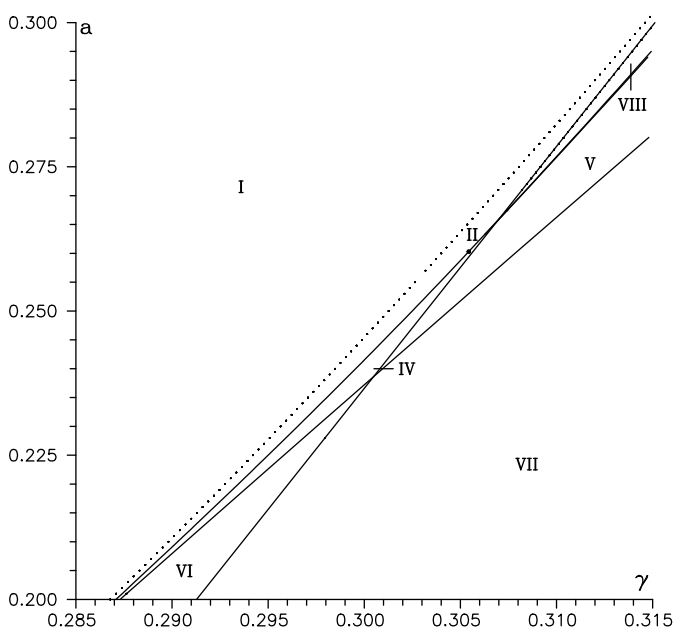

Figure 17. Regions of existence of the ferroelectric phase (fragment). $\omega=0.1$. The filled circle indicates the point where the curve of tricritical points forks into the curve of critical points and the curve of branchpoints.

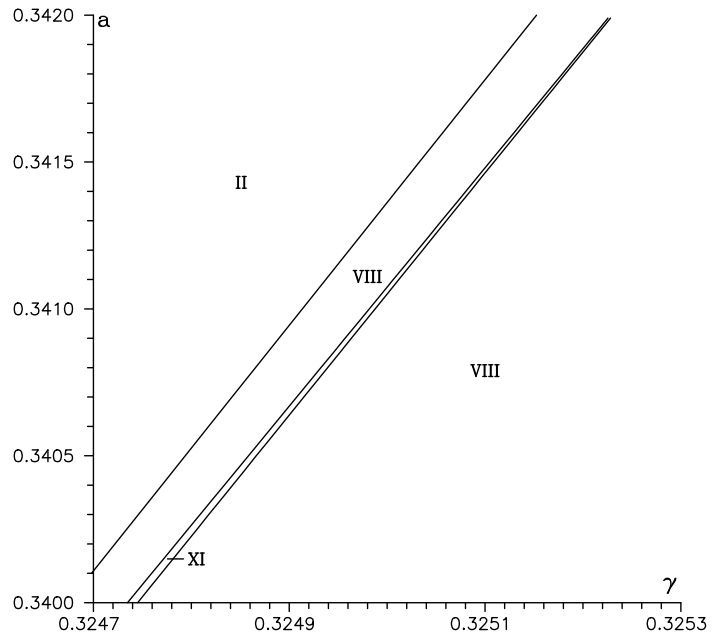

Figure 18. Regions of existence of the ferroelectric phase (fragment). $\omega=0.1$. 


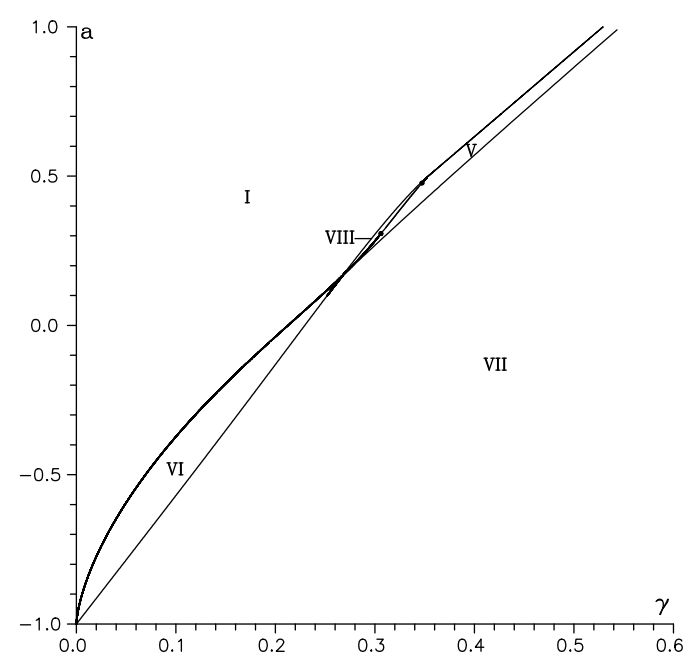

Figure 19. Regions of existence of the ferroelectric phase. $\omega=0.18$.

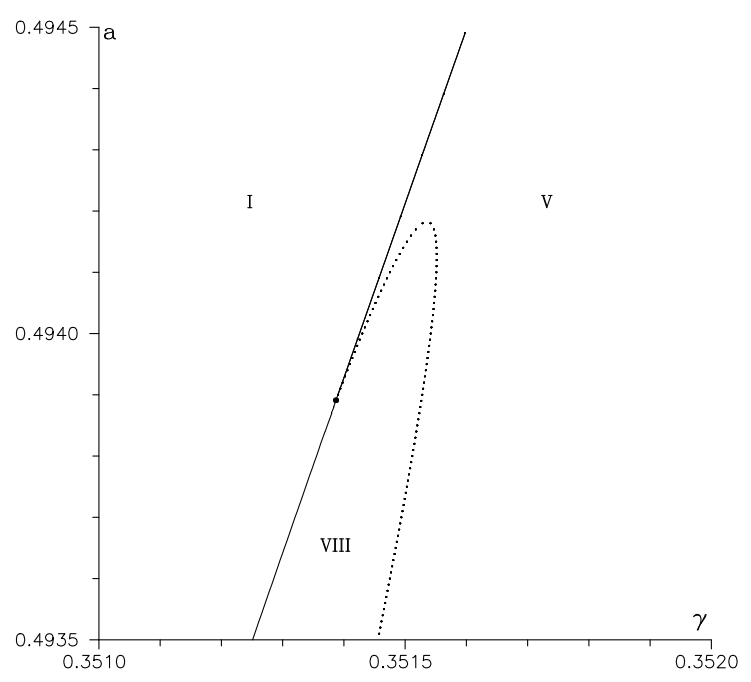

Figure 20. Regions of existence of the ferroelectric phase (fragment). $\omega=0.18$. Dotted line is the upper part of the curve of tricritical points.

( $a \approx 0.3433, \gamma \approx 0.3255, t \approx 0.0484$ ) cutting off a long spit from it (figure 18). This is region XI with two low-temperature first-order phase transitions and one second-order phase transition.

Further, the curve of maxima for the first-order phase transitions cuts off narrow strips from regions IX, V, and VII. These strips are regions XII, XIII, and XIV, respectively, where, in addition, two close low-temperature first-order phase transitions appear. In figure 17 these regions are not seen because they are too narrow.

As one can see, at a sufficiently small value of $\omega$ the diagram is richer than at $\omega=0$ but if the value of $\omega$ exceeds some number, the regions of the diagram disappear one after another and the diagram becomes poorer. Region III disappears firstly. In figure 17 it looks like a short curve segment at the beginning in the branchpoint. It is region XII that disappears the next, and, at $\omega=2^{-5 / 2}$, region $\mathrm{X}$ becomes a point.

\section{Conclusions}

Hence, we performed a complete analysis of phase transitions in the Mitsui model (without and with transverse field $\Omega$ ) in the mean field approximation. Some results concerning the phase diagram of the Mitsui model were obtained earlier [5, 12, 13] but they were incomplete (for the $\Omega=0$ case) or partial (for the $\Omega \neq 0$ case).

In the $\Omega=0$ case, we derived an analytical expression for tricritical temperature and the condition of its existence. In this case, there are seven regions in the plane $(a, \gamma)$ that correspond to seven different types of behavior of order parameters. At sufficiently small but nonzero $\Omega$ their number doubles. With $\Omega$ increasing these regions change their form and shift in the $(a, \gamma)$-plane. Starting with certain value of $\Omega$ the number of regions decrease and at $\omega \geqslant 1 / 2$ only the region without phase transitions remains.

At nonzero $\Omega$, second-order phase transitions are possible at zero temperature, which is not possible at $\Omega=0$. The maximal number of phase transitions is four and five at $\Omega=0$ and at $\Omega \neq 0$, respectively. 

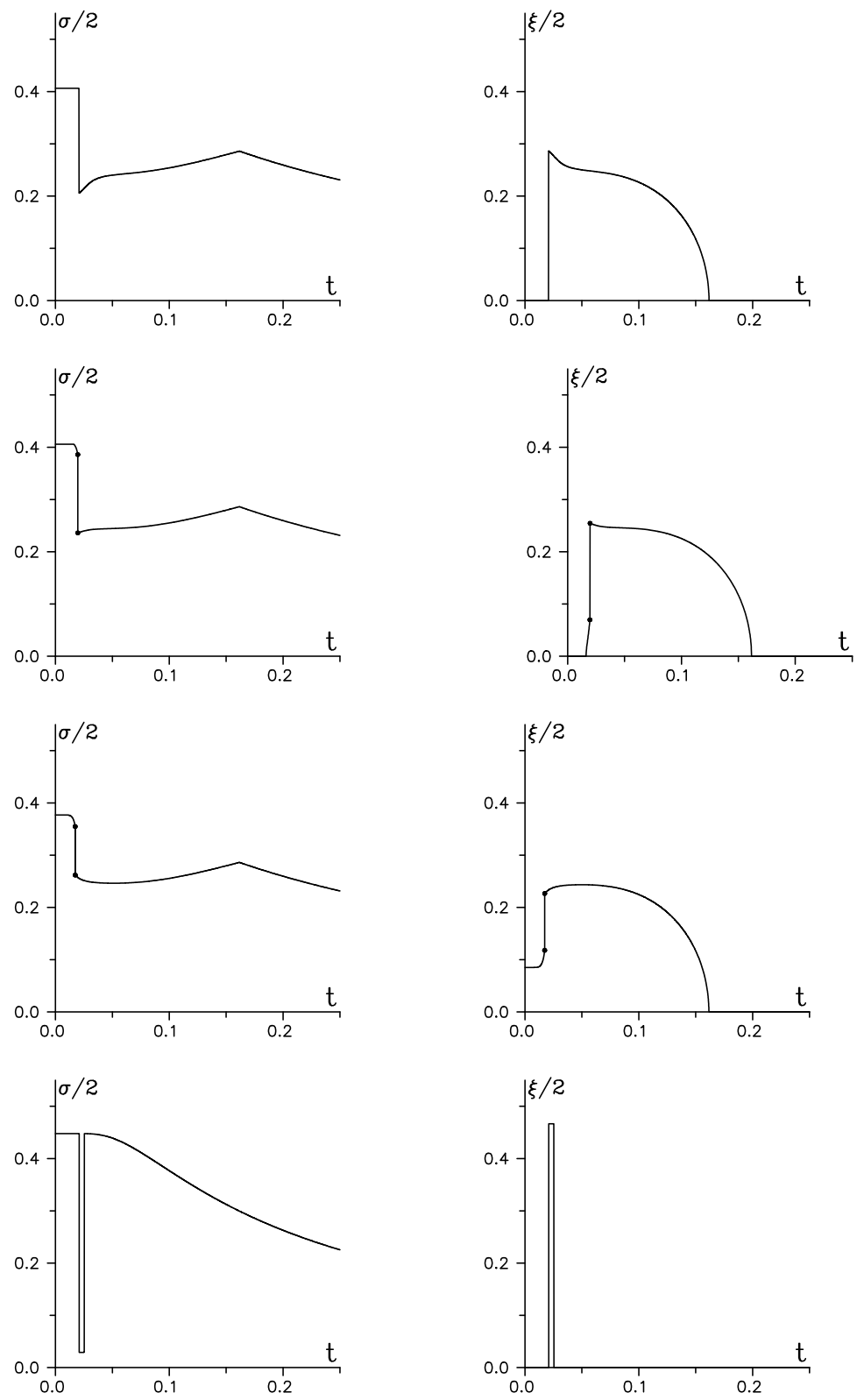

Figure 21. Ferroelectric and antiferroelectric order parameters for the regions of existence of ferroelectric phase. $\omega=0.1$. Only thermodynamically stable states are depicted. From top to bottom: VIII) $a=0.65, \gamma=0.4035$; IX) $a=0.66, \gamma=0.4065$; X) $a=0.667, \gamma=0.4085$; and $\mathrm{XIV}) a=0.2, \gamma=0.2912765$. The first-order phase transition within the ferroelectric phase is shown by filled circles.

\section{Acknowledgements}

The author is grateful to Prof. I. Stasyuk, Prof. R. Levitskii, Dr. T. Verkholyak and Dr. O. Danyliv for useful discussions. 


\section{References}

1. Mitsui T., Phys. Rev., 1958, 111, 1529; doi 10.1103/PhysRev.111.1259.

2. Žekš B., Shukla G.G., Blinc R., Phys. Rev. B, 1971, 3, 2306; doi 10.1103/PhysRevB.3.2306.

3. Levitskii R.R., Zachek I.R., Verkholyak T.M., and Moina A.P., Phys. Rev. B, 2003, 67, 174112; doi 10.1103 /PhysRevB.67.174112.

4. Levitskii R.R., Andrusyk A.Ya., Zachek I.R., Condens. Matter Phys., 2010, 13, 13705.

5. Levitskii R.R., Zachek I.R., Andrusyk A.Ya., J. Phys. Stud., 2010, 14, 3701.

6. Stasyuk I.V., Velychko O.V., Ferroelectrics, 2005, 316, 511; doi 10.1080/00150190590963138.

7. Levitskii R.R., Andrusyk A.Ya., Preprint of the Institute for Condensed Matter Physics, ICMP-1103U, Lviv, 2011 (in Ukrainian).

8. Levitskii R.R., Andrusyk A.Ya., Preprint of the Institute for Condensed Matter Physics, ICMP-0513U, Lviv, 2005 (in Ukrainian).

9. Levitskii R.R., Moina A.P., Andrusyk A.Ya., Slivka A.G., Kedyulich V.M., J. Phys. Stud., 2008, 12, 2603.

10. Danyliv O.D., Physica C, 1998, 309, 303; doi:10.1016/S0921-4534(98)00597-8.

11. Dublenych Yu.I., Preprint of the Institute for Condensed Matter Physics, ICMP-01-01U, Lviv, 2001 (in Ukrainian).

12. Vaks V.G., Introduction to the Microscopic Theory of Ferroelectrics. Nauka, Moscow, 1973 (in Russian).

13. Levitskii R.R., Verkholyak T.M., Kutnii I.V., Hil I.G., Preprint of the Institute for Condensed Matter Physics, ICMP-01-11U, Lviv, 2001 (in Ukrainian); Preprint arXiv:cond-mat/0106351, 2001.

\section{Фазові переходи в моделі Міцуі}

\section{Ю.І. Дубленич}

Інститут фізики конденсованих систем НАН України, вул. І. Свєнціцького, 1, 79011 Львів, Україна

В роботі в наближенні середнього поля досліджено фазові переходи в моделі Міцуі без поздовжнього поля, проте з поперечним полем. Встановлено взаємооднозначну залежність між такою моделлю та двопідґратковою моделлю типу Ізінга з поздовжнім і поперечним полями. Побудовано фазові діаграми та діаграми областей існування сегнетофази. Для випадку $\Omega=0(\Omega-$ поперечне поле) одержано простий аналітичний вираз для трикритичної температури й умову існування трикритичної точки. Для $\Omega \neq 0$ записано системи рівнянь для трикритичної точки й умови її існування.

Ключові слова: фазовий перехід, сегнетофаза, модель Міцуі, трикритична точка 